Supporting Information

\title{
How Sugars Modify Caffeine Self-Association and Solubility: Resolving a Mechanism of Selective Hydrotropy
}

Ilan Shumilin, Christoph Allolio, and Daniel Harries* 


\section{S1. Absorbance spectra of caffeine}

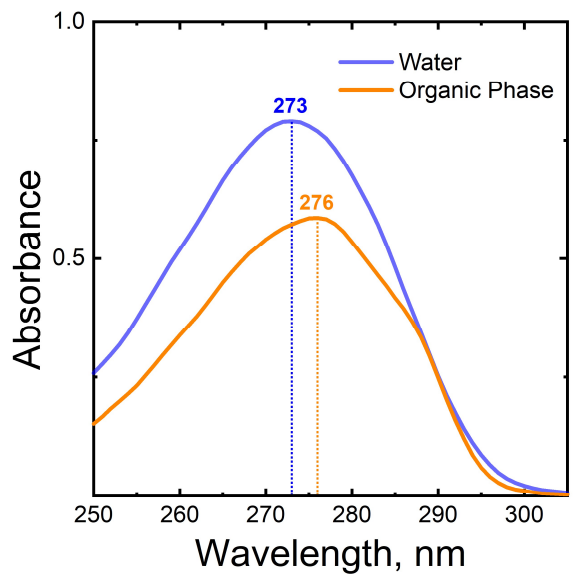

Figure S1: Absorbance spectra of caffeine, showing an adsorption peaks at $273 \mathrm{~nm}$ and $276 \mathrm{~nm}$ for aqueous and organic (isooctane:chloroform, 9:1, v/v) phases, respectively. Caffeine concentrations are $812 \mu \mathrm{M}$ and $52 \mu \mathrm{M}$ in aqueous and organic phases, respectively.

\section{S2. Chemical structure of materials}

Figure S2 shows the molecular structure of caffeine and sugars.

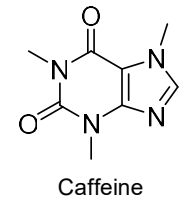<smiles>OC[C@@H]1O[C@H](O[C@@H]2O[C@H](CO)[C@H](O)[C@H]2O)[C@@H](O)[C@@H](O)[C@H]1O</smiles>

Sucrose

$\alpha$-D-glucopyranosyl-(1 $\rightarrow 2)-\beta$-D-fructofuranoside

(nonreducing)<smiles>OC[C@H]1O[C@H](O)[C@H](O)[C@H](O)[C@H]1O</smiles>

(D)-Glucose

(reducing)<smiles>OC[C@H]1O[C@](O)(CO)[C@@H](O)[C@H]1O</smiles>

$\mathrm{OH} \quad \mathrm{OH}$<smiles>OC[C@H]1O[C@@H](O[C@H]2O[C@H](CO)[C@H](O)[C@@H](O)[C@H]2O)[C@H](O)[C@@H](O)[C@@H]1O</smiles>

$\alpha$-D-glucopyranosyl-(1 $\rightarrow 1)$-a-D-glucopyranoside

(nonreducing)

Figure S2: Chemical structure of caffeine and sugars. For reducing sugars, equilibrium exists between anomeric states. Chemical structures were plotted using ChemDraw. 


\section{S3. Details of the isodesmic model of solute association}

For isodesmic binding, molecules of type $M$ can form oligomers of any size $n$ by following a series of reactions of monomer addition of the type:

$$
\begin{gathered}
M+M \rightarrow M_{2} \\
M+M_{2} \rightarrow M_{3} \\
\vdots \\
M+M_{n-1} \rightarrow M_{n}
\end{gathered}
$$

In the isodesmic model, the equilibrium constants for all these reactions are equal, and therefore there is effectively only one "isodesmic equilibrium constant", $K$. The equilibrium constants are defined by:

$$
\begin{gathered}
K=\frac{c_{M_{2}}}{c_{M}^{2}} \\
K=\frac{c_{M_{3}}}{c_{M_{2}} c_{M}}=\frac{c_{M_{3}}}{K c_{M}^{3}} \\
\vdots \\
K=\frac{c_{M_{n}}}{c_{M_{n-1}} c_{M}}
\end{gathered}
$$

Thus, the concentration of any aggregate size can be calculated by knowing the monomer concentration and isodesmic constant:

$$
c_{M_{n}}=K^{n-1} c_{M}^{n}
$$

Denoting $c_{i}^{\circ}$ as the total concentration of molecules $M$ in solution $i$ ( $i=a q$ in aqueous solutions), $c_{a q}^{o}$, is simply:

So that combining Equations 3.2 and 3.4 we find:

$$
c_{a q}^{o}=c_{M}+2 c_{M_{2}}+\cdots+n c_{M_{n}}
$$

$$
c_{a q}^{o}=c_{M}\left[1+2 K c_{M}+3 K^{2} c_{M}^{2}+4 K^{3} c_{M}^{3}+\cdots+n K^{n-1} c_{M}^{n-1}\right]=\frac{c_{M}}{\left(1-K c_{M}\right)^{2}}
$$

The concentration of the monomer is then obtained by solving Equation 3.5 as a quadratic equation:

$$
c_{M}=\frac{4 c_{a q}^{o}}{\left[1+\left(4 K c_{a q}^{o}+1\right)^{1 / 2}\right]^{2}}
$$

\section{S4. Using partition experiments to determine the isodesmic constant}

At equilibrium, the chemical potential of caffeine molecules is equal in both the aqueous and organic phases for all species (monomers and oligomers of all sizes). Therefore, the condition for equilibrium between the two phases can be expressed using the chemical potential of the monomer:

$$
\mu_{M, a q}=\mu_{M, o r g}
$$

which can also be written in terms of the corresponding concentrations in the ideal solution limit:

$$
\mu_{M, a q}^{\circ}+R T \ln c_{M, a q}=\mu_{M, o r g}^{\circ}+R T \ln c_{M, o r g}
$$

Therefore, by rearranging Equation 4.2, the partitioning of the monomer between both phases can be related to the chemical potentials through:

$$
\frac{c_{M, a q}}{c_{M, o r g}}=\exp \left(\frac{\mu_{M, o r g}^{\circ}-\mu_{M, a q}^{\circ}}{R T}\right)
$$

Combining Equations 3.6 and 4.3, we then have:

$$
\frac{c_{M, a q}}{c_{M, o r g}}=\frac{4 c_{a q}^{\circ}}{c_{M, o r g}\left[1+\left(4 K c_{a q}^{\circ}+1\right)^{1 / 2}\right]^{2}}=\exp \left(\frac{\mu_{M, o r g}^{\circ}-\mu_{M, a q}^{\circ}}{R T}\right)
$$

This can also be re-expressed as:

$$
\frac{c_{M, o r g}}{c_{a q}^{\circ}}=\frac{c_{M, o r g}}{c_{M, a q}} \cdot \frac{4}{\left[1+\left(4 K c_{a q}^{\circ}+1\right)^{1 / 2}\right]^{2}}
$$

The partition coefficient, $P$, is defined as:

$$
P=\frac{c_{o r g}^{\circ}}{c_{a q}^{\circ}}
$$

Now we note that since at infinite dilution all caffeine is in the monomeric state, the partition coefficient at infinite dilution is simply:

$$
P_{\text {inf }}=\lim _{c_{a q}^{o} \rightarrow 0} P=\lim _{c_{a q} \rightarrow 0} \frac{c_{o r g}^{\circ}}{c_{a q}^{\circ}}=\frac{c_{M, o r g}}{c_{M, a q}}
$$


Water solubilizes caffeine much better compared to the organic phase we used. Therefore, by performing partitioning experiments of caffeine at very low concentrations, specifically in the range of $100-400 \mu \mathrm{M}$ in the organic phase, we can neglect any caffeine oligomerization in the organic phase. Hence, $c_{M, o r g}$ is the total and monomeric concentration of caffeine in the organic phase. Therefore, we can express Equation 4.5 in terms of the partition coefficients as:

$$
P=P_{\text {inf }} \cdot \frac{4}{\left[1+\left(4 K c_{a q}^{\circ}+1\right)^{1 / 2}\right]^{2}}
$$

The partition of caffeine at equilibrium is determined experimentally by absorbance, while caffeine partitioning at infinite dilution, $P_{\text {inf }}$, is determined as the intercept of the curve fitted to the plots in Figure S3.

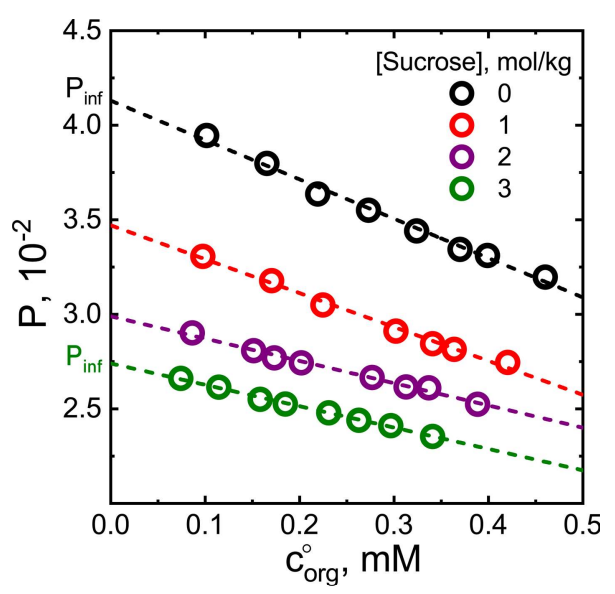

Figure S3. Partition coefficient of caffeine (as defined in Equation 4.6) vs caffeine concentration in the organic phase at different sucrose concentrations in the aqueous phase. The dashed lines represent linear fits to several sets of caffeine partitioning at different sucrose concentrations, where the partition coefficient at infinite dilution $\left(P_{\mathrm{inf}}\right)$ represents the value of the intercept (as labelled for example in black and green for pure water and for $3 \mathrm{~mol} / \mathrm{kg}$ sucrose, respectively).

\section{S5. Caffeine chemical potential at saturation}

We can distinguish three possible states for caffeine, with corresponding chemical potentials: (i) free monomer in solution with $\mu_{M}$ (ii) aggregate composed of $n$ caffeine molecules with $\mu_{M_{n}}$ and (iii) insoluble solid caffeine with $\mu_{S}$.

At saturation, the condition for equilibrium between monomers and oligomers requires that:

$$
\mu_{M_{n}, s a t}=n \mu_{M, s a t}
$$

While the equilibrium between solid and solvated monomers requires also that:

$$
\mu_{S}=\mu_{M, s a t}=\mu_{M}^{\circ}+R T \ln c_{M, s a t}
$$

where $\mu_{M}^{\circ}$ is the standard chemical potential of a monomer. Because the chemical potential of any aggregate of size $n$ at saturation is:

we can combine Equations 5.1, 5.2 and 5.3 to get:

$$
\mu_{M_{n}, s a t}=\mu_{M_{n}}^{\circ}+R T \ln c_{M_{n}, s a t}
$$

$$
\mu_{M_{n}}^{\circ}-n \mu_{S}=-R T \ln c_{M_{n}, s a t}
$$

Specifically, for the monomer, $n=1$, so that

$$
\mu_{M}^{\circ}-\mu_{S}=-R T \ln c_{M, s a t}
$$




\section{S6. Deriving isodesmic expressions for tip and stack in caffeine oligomers}

Since caffeine oligomers can be dissected into stack and tip contributions, and noticing that in the isodesmic model the two tips contributions amount to the chemical potential of a monomer, we can write the oligomer's chemical potential as composed of two terms:

$$
\mu_{M_{n}}^{\circ}=\mu_{M}^{\circ}+(n-1) \mu_{\text {stack }}^{\circ}
$$

Using Equations 5.4, 5.5, 6.1 and 3.3 at saturation, we have:

$$
\mu_{\text {stack,sat }}^{\circ}-\mu_{S}=-R T \ln K c_{M, s a t}
$$

Note that the standard chemical potential of the tip is equal to that of the monomer, which can be calculated using Equation 5.2 . Alternatively the tip contribution can also be determined by the difference:

$$
\mu_{\text {tip }}^{\circ}=\mu_{M_{n}}^{\circ}-(n-1) \mu_{\text {stack }}^{\circ}
$$

\section{S7. Crystal structure of caffeine aggregates}

Figure S4 shows the known crystal structure of caffeine aggregates in both dehydrated ${ }^{1}$ and hydrated ${ }^{2}$ (with 0.8 equivalents of water) states. Both structures show a distinct columnar arrangement of caffeine stacks that are tightly packed next to each other, in a sideby-side arrangement.
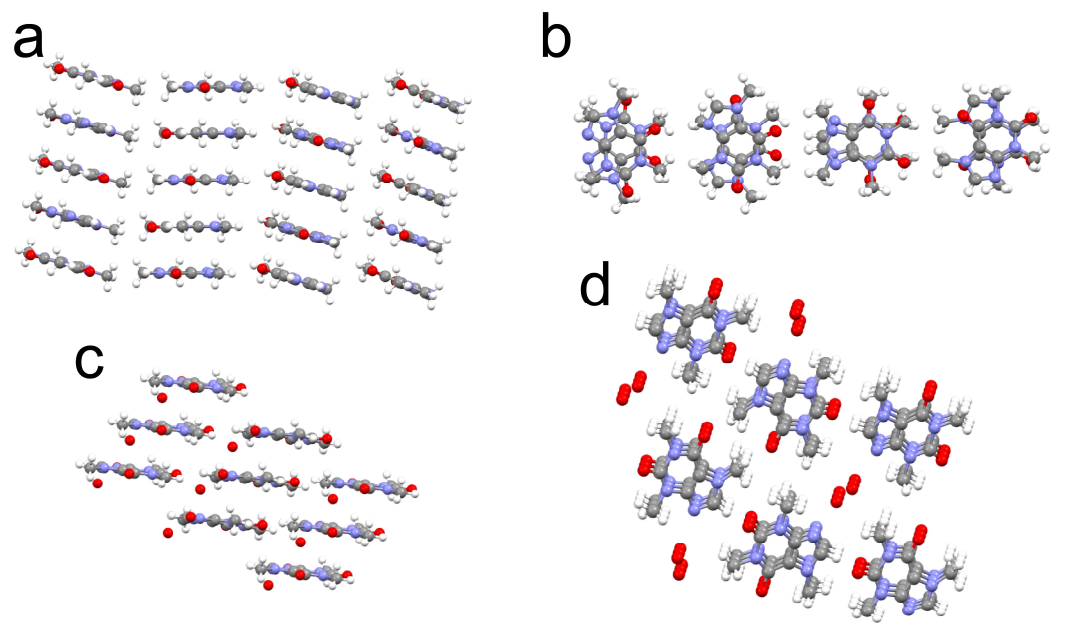

Figure S4. Caffeine crystal structure of $(a, b)$ anhydrous and $(c, d)$ hydrated with 1:0.8 caffeine to water mole ratio. Red dots in figures $\mathrm{c}$ and $\mathrm{d}$ represent water molecules. 


\section{S8. Depletion forces}

The change in free energy associated with aggregation into the crystal form of caffeine involves the burial of surfaces previously exposed to solution. Compared with the oligomeric state, most of the buried surface comes from adjoining side-by-side stacks' surfaces. In the presence of excluded solute, depletion forces arise. In the van 't Hoff limit, the free energy contribution to the depletion force is proportional to the change in excluded volume upon aggregation, $\Delta V_{e x}$, and to the osmotic pressure of solution, $\Pi$, via $\Delta G=\Pi \Delta V_{e x} \cdot{ }^{3-7}$ For simple depletants that act through excluded volume alone, the extent of excluded volume due to the stackexposed surfaces approximately scales as $\Delta V_{e x} \sim \sigma_{c} \bar{V}_{s}^{1 / 3}$, where $\sigma_{c}$ is the effective area of solvent exposed caffeine stack, $\bar{V}_{s}$ is sugar partial molar volume, and $\bar{V}_{s}^{1 / 3}$ is the sugar's linear dimension. Caffeine's accessible surface area was computed using the sasa command implemented in GROMACS, ${ }^{8}$ and sugar's partial molar volume was calculated from the density measurements (see section S13) using the standard method (see e.g. ref (9)). Specifically, the change in solvent-exposed area of caffeine stacks per monomer was estimated by subtracting the computed surface of caffeine monomer from the surface of the constrained octamer, and divided by 7 . Because the osmotic pressure varies linearly with sugar concentration, $m_{s}$, at the low concentration van't Hoff limit, $m_{s} \rightarrow 0$, the free energy change due to the depletion interaction then approximately scales as $\Delta G \sim m_{s} \sigma_{c} \bar{V}_{s}^{1 / 3}$. Figures S5a,b show the free energy change for both precipitation free energy and stack chemical potential versus the expected scaling dependence for the depletion interaction. We find that while sucrose, glucose, and fructose show similar scaling, trehalose acts more strongly than the other 3 sugars. This suggests that the effective excluded volume is larger for trehalose, and possibly depends on interactions beyond those predicted by its partial molar volume alone.
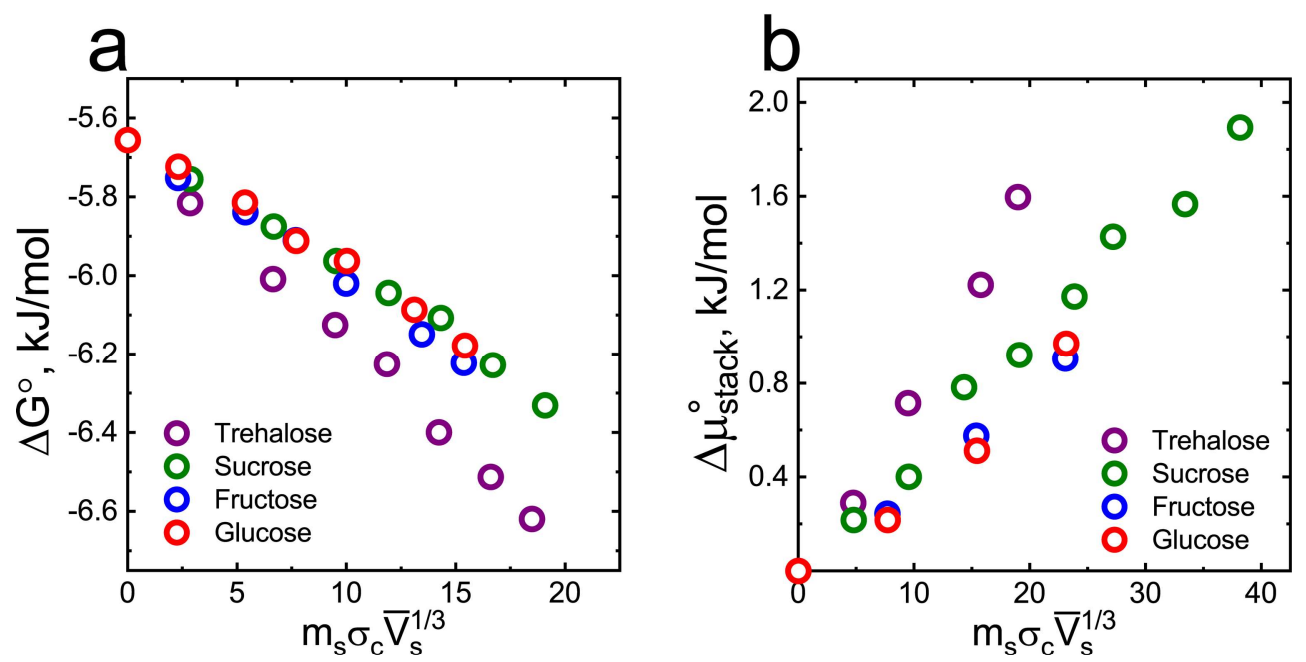

Figure S5. a) Caffeine precipitation free energy versus the product of sugar molality and estimated excluded volume change, $m_{s} \sigma_{c} \bar{V}_{s}^{1 / 3}$, for all examined sugars. b) The change in caffeine stack chemical potential upon sugar addition versus $m_{s} \sigma_{c} \bar{V}_{s}^{1 / 3}$, for different sugars.

\section{S9. Free energy of monomer association in experiments and simulations}

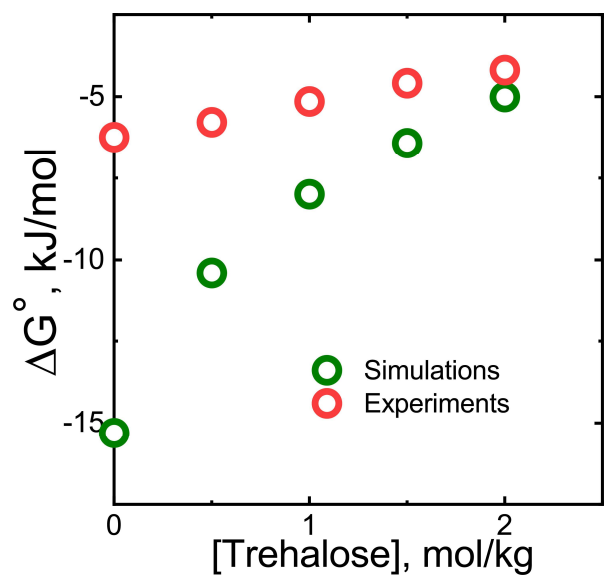

Figure S6. Values of $\Delta G^{o}$ for monomer addition to oligomers from experiments and from molecular dynamics simulations versus trehalose molal concentration. 


\section{S10. Kirkwood-Buff integrals and the link to preferential interactions}

We calculated the radial distribution functions, $g(r)$, from molecular dynamics simulations. The Kirkwood-Buff integrals, $G_{i j}$, are then calculated by integrating over the radial distribution functions: ${ }^{10,11}$

$$
G_{i j}=4 \pi \int\left(g_{i j}(r)-1\right) r^{2} d r
$$

The preferential hydration coefficient, $\Gamma_{W}$, can then be determined using:

$$
\Gamma_{W}=55.6\left(G_{W C}-G_{C S}\right)
$$

where 55.6 is the molality of water, and $W, C$, and $S$ represent water, caffeine, and sugar, respectively. The preferential interaction coefficient with sugar, $\Gamma_{S}$, is derived using the known relation ${ }^{12,13}$ :

$$
\Gamma_{S}=-\frac{m_{s}}{55.6} \Gamma_{W}
$$

where $m_{s}$ is the molal concentration of sugar.

\section{S11. Linking interactions to changes in free energy}

\section{S11.1 Potential of mean force}

Using the first peak of the radial distribution function $\mathrm{g}(\mathrm{r})$ for trehalose around monomeric caffeine (see Figure $3 \mathrm{c}$, top panel), the Potential of Mean Force (PMF) is determined as $P M F=-R T \ln g(\mathrm{r}) \cong-5.05 \mathrm{~kJ} / \mathrm{mol}$. Thus, the change in $\Delta G^{o}$ of caffeine oligomerization, $\Delta \Delta G^{\circ}$, can be approximated as the product of PMF and $\Delta \Gamma_{S}$, resulting in $\Delta \Delta G^{\circ} \cong 5.50 \mathrm{~kJ} / \mathrm{mol}$.

\section{S11.2 Change in hydrogen bonding upon oligomerization in simulation}

We determined the average number of hydrogen bonds formed between all components in solution for a simulation of pure water (4067 water molecules) and $1 \mathrm{~mol} / \mathrm{kg}$ trehalose solution (74 trehalose molecules). Both simulations were repeated for monomeric caffeine and a restrained oligomer with $n=8$. Table $\mathrm{S} 1$ shows the difference in the average number of $\mathrm{H}$-bonds formed between

\begin{tabular}{|c|c|c|c|c|c|c|c|}
\hline \multirow[b]{2}{*}{$\begin{array}{l}\text { H-Bond } \\
\text { type }\end{array}$} & \multicolumn{3}{|c|}{$\begin{array}{l}\text { Trehalose Concentration } \\
\qquad 0 \mathrm{~mol} / \mathrm{kg}\end{array}$} & \multicolumn{3}{|c|}{$\begin{array}{l}\text { Trehalose Concentration } \\
\qquad 1 \mathrm{~mol} / \mathrm{kg}\end{array}$} & \multirow[b]{2}{*}{$\Delta \Delta$ Oligomerization $^{[\mathrm{d}]}$} \\
\hline & Monomer ${ }^{[a]}$ & Oligomer $^{[\mathrm{b}]}$ & $\Delta$ Oligomerization $^{[\mathrm{c}]}$ & Monomer ${ }^{[a]}$ & Oligomer $^{[b]}$ & $\Delta$ Oligomerization $^{[c]}$ & \\
\hline WW & $-4.2 \pm 0.1$ & $-23.2 \pm 0.1$ & $1.5 \pm 0.1$ & $-2.6 \pm 0.5$ & $-17.4 \pm 0.5$ & $0.5 \pm 0.6$ & $-1.0 \pm 0.6$ \\
\hline WC & $4.06^{*}$ & $25.15 \pm 0.01$ & $-1.05 \pm 0.01$ & $2.70 \pm 0.01$ & $21.52 \pm 0.01$ & $-0.01 \pm 0.01$ & $1.04 \pm 0.01$ \\
\hline WT & - & - & - & $-2.7 \pm 0.3$ & $-5.97 \pm 0.3$ & $2.2 \pm 0.4$ & $2.2 \pm 0.4$ \\
\hline $\mathrm{TC}$ & - & - & - & $1.14 \pm 0.01$ & $2.88 \pm 0.01$ & $-0.89 \pm 0.01$ & $-0.89 \pm 0.01$ \\
\hline $\mathrm{TT}$ & - & - & - & $0.00 \pm 0.08$ & $-0.25 \pm 0.07$ & $-0.04 \pm 0.01$ & $-0.04 \pm 0.01$ \\
\hline Total & $-0.2 \pm 0.1$ & $2.0 \pm 0.1$ & $0.5 \pm 0.1$ & $-1.5 \pm 0.6$ & $0.8 \pm 0.6$ & $1.8 \pm 0.7$ & $1.3 \pm 0.7$ \\
\hline
\end{tabular}
components in solutions bathing caffeine and that of a solution without caffeine, with equal trehalose concentrations.

Table S1. Difference in the average number of hydrogen bonds formed between binary combinations of caffeine (C), trehalose (T), and water (W).

[a] Results for simulation with a single caffeine molecule. [b] Results for simulation with a restrained oligomer ( $\mathrm{n}=8$ ). [c] Change upon oligomerization. [d] The difference in hydrogen bonding upon transfer from water to $1 \mathrm{~mol} / \mathrm{kg}$ trehalose solution. $\left.{ }^{\star}\right]$ Value with error significantly smaller than that of the value itself. 


\section{S12. Monomer fraction and solubility}

We calculated monomeric caffeine concentrations using Equation S3.6 for different sugar concentrations. Table S2 shows a compilation of reported fraction and solubility of monomeric caffeine in aqueous solutions of sugars from our own work as well as in, salts and denaturants such as urea and guanidinium- $\mathrm{HCl}$ determined in the work of Al-Maaieh et al ${ }^{14}$ and Cesaro et $a l^{15}$.

Table S2. The fraction and total solubility of monomeric caffeine in solution.

\begin{tabular}{|c|c|c|c|c|}
\hline Cosolute & Cosolute Conc. [M] & Monomer Fraction in soluble species & Monomer Solubility [M] & Caffeine Solubility [M] \\
\hline Water ${ }^{[a]}$ & & 0.334 & 0.0336 & 0.1005 \\
\hline \multirow[t]{4}{*}{ Trehalose $\mathrm{e}^{[\mathrm{a}]}$} & 0.45 & 0.390 & 0.0359 & 0.092 \\
\hline & 0.83 & 0.468 & 0.0391 & 0.0835 \\
\hline & 1.23 & 0.552 & 0.0399 & 0.0722 \\
\hline & 1.40 & 0.607 & 0.0403 & 0.0664 \\
\hline \multirow[t]{8}{*}{ Sucrose ${ }^{[a]}$} & 0.45 & 0.376 & 0.0356 & 0.0946 \\
\hline & 0.82 & 0.411 & 0.0365 & 0.0887 \\
\hline & 1.14 & 0.480 & 0.0397 & 0.0828 \\
\hline & 1.40 & 0.504 & 0.0387 & 0.0769 \\
\hline & 1.63 & 0.544 & 0.0387 & 0.071 \\
\hline & 1.77 & 0.583 & 0.0390 & 0.0669 \\
\hline & 2.00 & 0.603 & 0.0357 & 0.0592 \\
\hline & 2.15 & 0.647 & 0.0345 & 0.0533 \\
\hline \multirow[t]{3}{*}{ Fructose ${ }^{[a]}$} & 0.90 & 0.381 & 0.0344 & 0.0902 \\
\hline & 1.63 & 0.443 & 0.0354 & 0.0799 \\
\hline & 2.24 & 0.501 & 0.0348 & 0.0696 \\
\hline \multirow[t]{3}{*}{ Glucose ${ }^{[a]}$} & 0.90 & 0.376 & 0.0343 & 0.0911 \\
\hline & 1.63 & 0.432 & 0.0352 & 0.0816 \\
\hline & 2.23 & 0.512 & 0.0369 & 0.0721 \\
\hline Water $\left[r^{[b]}\right.$ & & 0.327 & 0.0350 & 0.1070 \\
\hline \multirow[t]{3}{*}{$\mathrm{Na}_{2} \mathrm{SO}_{4}^{[b]}$} & 0.25 & 0.373 & 0.0247 & 0.0662 \\
\hline & 0.5 & 0.429 & 0.0175 & 0.0408 \\
\hline & 1.0 & 0.546 & 0.0099 & 0.0181 \\
\hline \multirow[t]{3}{*}{$\mathrm{NaClO}_{4}{ }^{[\mathrm{b}]}$} & 0.25 & 0.306 & 0.0518 & 0.1693 \\
\hline & 0.5 & 0.271 & 0.0630 & 0.2325 \\
\hline & 1.0 & 0.233 & 0.0898 & 0.3854 \\
\hline $\mathrm{NaSCN}^{[b]}$ & 1.0 & 0.243 & 0.0776 & 0.3193 \\
\hline $\mathrm{NaCl} I^{[b]}$ & 1.0 & 0.383 & 0.0273 & 0.0713 \\
\hline $\mathrm{NaBr} r^{[b]}$ & 1.0 & 0.348 & 0.0390 & 0.1121 \\
\hline Water ${ }^{[c]}$ & & 0.373 & 0.0401 & 0.1075 \\
\hline $\mathrm{KCl}]^{[c]}$ & 1.0 & 0.360 & 0.0270 & 0.075 \\
\hline Urea ${ }^{[\mathrm{c}]}$ & 1.0 & 0.134 & 0.0654 & 0.4881 \\
\hline Gu-HCl[c] & 1.0 & 0.191 & 0.0880 & 0.4607 \\
\hline
\end{tabular}

[a] This work. [b] Work by Al-Maaieh et al. [c] Work by Cesaro et al. 


\section{S13. Caffeine saturation concentration}

Using solubility data (Figure 1a) and Equations 3.3, 3.6, we calculated and present the monomer, dimer, trimer, and octamer concentrations at caffeine saturation for different sugar content. Figure S7a shows the solubility of these species in $1 \mathrm{~mol} / \mathrm{kg} \mathrm{sugar}$ solutions, $c_{M_{n}}$ relative to the solubility in pure water, $c_{M_{n}, 0}$. Similarly, Figure S7b shows the relative solubility normalized by the partial molar volume of sugars $\left(\bar{V}_{s}\right)$. The volumes of the sugars were calculated from density measurements (see section S15). These volumes are presented in the inset of Figure $\mathrm{S} 7 \mathrm{~b}$.
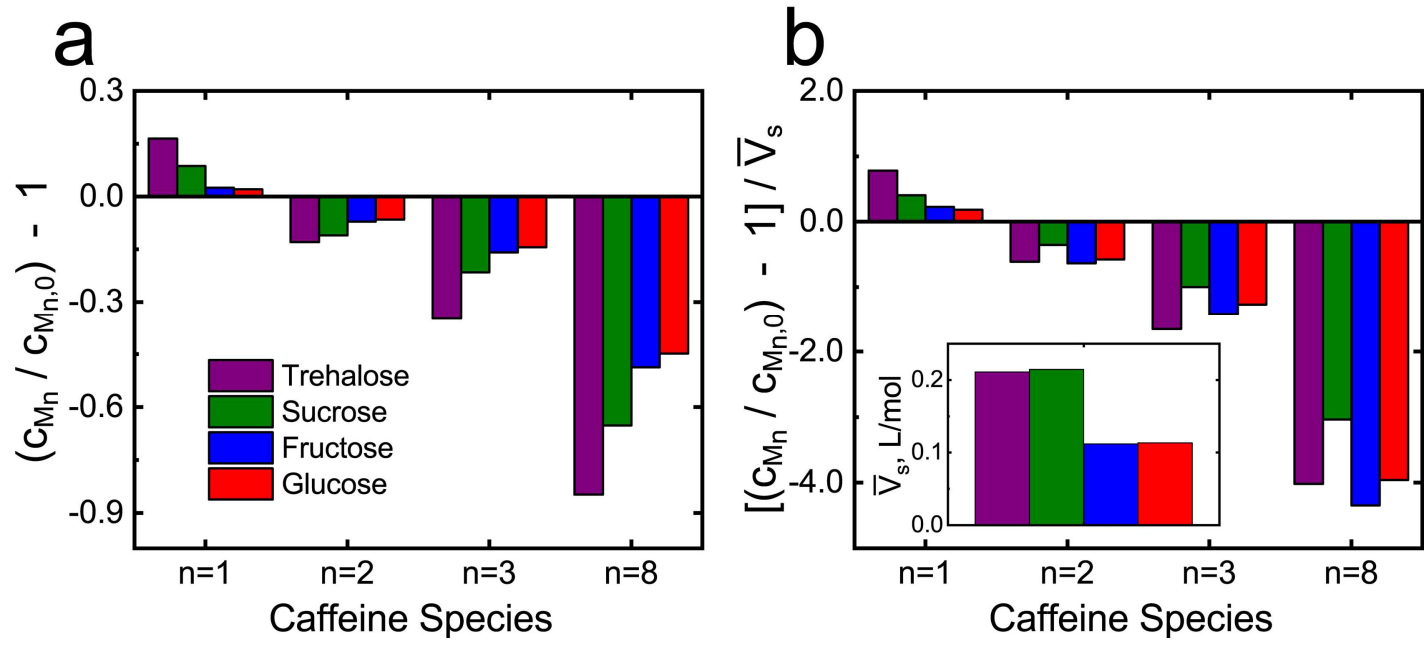

Figure S7. a) Saturation concentration of monomer, dimer, trimer, and octamer in $1 \mathrm{~mol} / \mathrm{kg}$ sugar solutions relative to the solubility in pure water. Data is presented as the difference from unity. b) Relative concentration scaled by the partial molar volume of each sugar. The inset shows the calculated sugar partial molar volumes. 


\section{S14. Convergence of simulations}

To verify that the timescales used for analyzing caffeine self-association were sufficiently long to sample the equilibrium state, we compared the probability distribution of caffeine oligomers using different segments of two independent simulation runs, where $\mathrm{N}=8$ caffeine molecules dissolved in water $\left(c_{a q}^{o}=0.109 \mathrm{~mol} / \mathrm{kg}\right.$ ), with a total duration of $1.2 \mu \mathrm{s}$ and $0.6 \mu \mathrm{s}$. To allow initial equilibration, we only used the second half of each simulation for analysis: a 300ns segment of the short simulation, and a total of 600ns of the long simulation, that for comparison were further divided into two sequential segments each lasting 300ns. Figure S8a shows the average oligomerization free energy for each oligomer size (black squares), with standard errors from the 3 simulation segments shown as error bars. Also shown is the total averaged oligomerization free energy of all oligomer sizes over the three segments, represented as an orange bar with width corresponding to the standard error, Figure S8a. We find that the averaged free energy of monomer addition to oligomers of sizes $n=1$ to $n=6$ were within or close to the standard errors of the total average free energy. This indicates that a timescale of $\sim 1 \mu \mathrm{s}$, as used for analyzed simulations, is sufficiently long for sampling converged data. This result is further supported by counting dissociation and aggregation events, which was performed by comparing the connectivity between frames at a cutoff of $6.0 \AA$. The increased cutoff was chosen to avoid counting structural fluctuations. We found 780 dissociation (and a similar number of associations) events per microsecond. The frequent transition events between oligomer sizes permits an equilibrated distribution of caffeine oligomers within our simulation timescale.

The finite size of simulations is known to have a potential impact on molecular clustering equilibria in solution. ${ }^{16}$ To assess the extent to which the finite size of our simulations affect the resulting isodesmic association constant in the presence of sugar, we repeated the simulation of caffeine in the presence of $0.5 \mathrm{~mol} / \mathrm{kg}$ sugar, but with only $\mathrm{N}=4$ (rather than $\mathrm{N}=8$ ) caffeine molecules, thereby reducing the caffeine concentration by half. If inclusion or exclusion of sugar from the oligomerizing caffeine impacts the relevant chemical potential of sugar (i.e. the relevant bulk sugar activity), we should expect to see a difference in the resulting oligomerization free energy that would be concomitant to these bulk concentration changes. These changes should be strongest at small sugar concentrations, motivating our choice of $0.5 \mathrm{~mol} / \mathrm{kg}$ trehalose concentration. Figure S8b,c compares the oligomer concentrations in these two simulations and the resulting association free energy. We find a very slight decrease in the association free energy in the $\mathrm{N}=4$ system compared with $\mathrm{N}=8$. Although this difference is very close to statistical uncertainty, it may indicate a weak finite size effect; due to the exclusion of sugar from caffeine oligomers, the effective concentration of sugar in the bulk is higher for $\mathrm{N}=8$ compared with $\mathrm{N}=4$ due to the larger number of excluding caffeines. This lowers the impact of sugar on the free energy of caffeine association for $\mathrm{N}=4$, which is indeed what we observe: caffeine oligomerization free energy increases less in the $\mathrm{N}=4$ system.

Finally, we also tested the possible finite size effect for caffeine self-aggregation, comparing water and caffeine simulations with $\mathrm{N}=4$ and $\mathrm{N}=8$ (in absence of sugar). In this system, we find nearly identical association free energies within the respective errors (compare red and black symbols in Figure S8a). The over aggregative nature of the caffeine-caffeine interactions together with the possible finitesize effect do impact the population of largest oligomer size, but this does not modify the isodesmic constant up to $n=6$, and this result is independent of caffeine concentration (compare the $\mathrm{N}=8$ and $\mathrm{N}=4$ simulations). The largest aggregates (hollow symbols in Figure S8a), thus act as a reservoir that keeps the caffeine chemical potential constant, as determined by the same $\Delta G^{\circ}$ for monomers and other oligomers. Furthermore, even for the lowest sugar concentration $(0.5 \mathrm{~mol} / \mathrm{kg})$, the caffeine association free energy is equal for all oligomer sizes within sampling error (Figure S8b). Our finding support averaging over $n=1$ through $n=7$ clusters in the $N=8$ simulations.
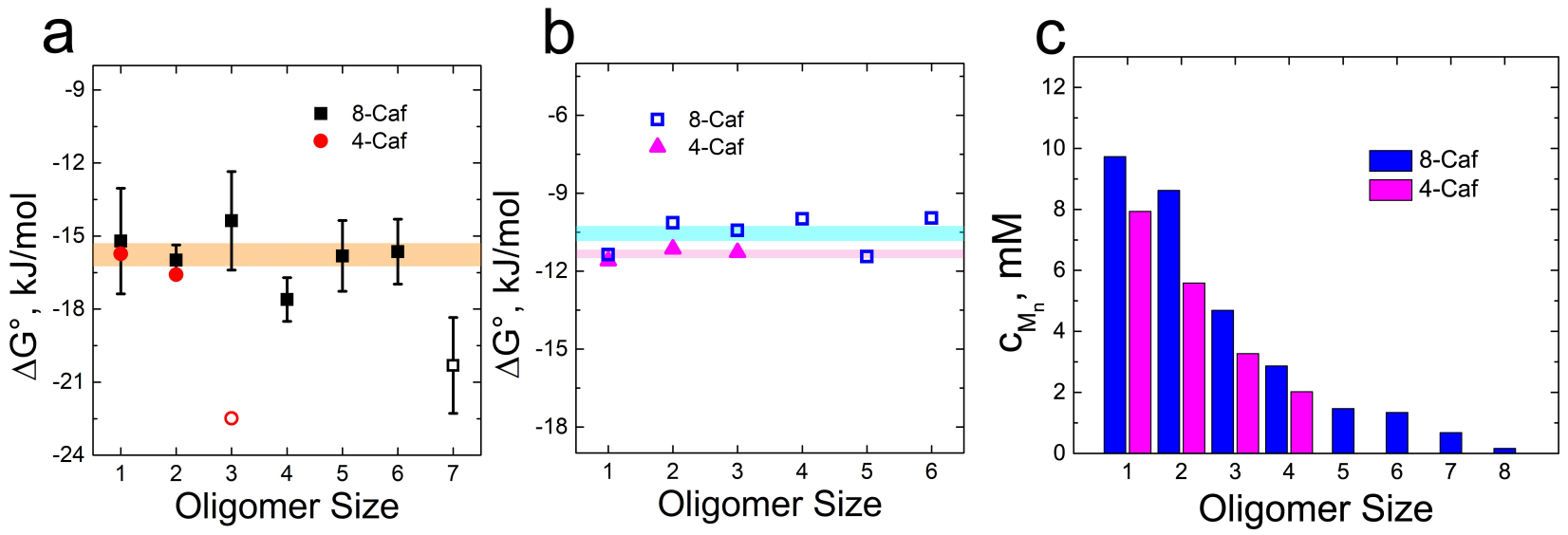

Figure S8. a) Average monomer to oligomer addition free energy (orange line) and average free energy per monomer addition to oligomers of sizes $n=1$ to $n=6$ (black squares) for a total of $900 n$ s of simulation trajectories in pure water, with a total of $N=8$ caffeine molecules. The standard error of total free energy is represented by the width of the orange line, whereas the values and error bars of the black squares represent the average and standard error of three segments of simulation runs each lasting 300ns. Red circles represent oligomerization free energy in simulation of $\mathrm{N}=4$ caffeine molecules. b) Average monomer to oligomer addition free energy of (i) sizes $\mathrm{n}=1$ to $\mathrm{n}=6$ in simulation of $\mathrm{N}=8$ caffeine molecules (blue squares) and (ii) sizes $n=1$ to $n=3$ in simulation of $N=4$ caffeine molecules (pink triangles). In both simulations caffeine is bathed in $0.5 \mathrm{~mol} / \mathrm{kg}$ trehalose solution. The standard error of total free energy in both simulations is represented by the width of the blue and pink lines, which correspond to the simulations of $\mathrm{N}=8$ and $\mathrm{N}=4$ caffeine molecules, respectively. c) Average concentration of oligomers in simulation of $\mathrm{N}=8$ caffeine molecules in $0.5 \mathrm{~mol} / \mathrm{kg}$ trehalose solution (blue) and $\mathrm{N}=4$ caffeine molecules in $0.5 \mathrm{~mol} / \mathrm{kg}$ trehalose solution (pink). 


\section{S15. Sugar solution densities}

Sugar solution densities were determined by a DMA 5000 M (Anton Paar) densitometer. Figure S9 shows the density of aqueous sugar solutions at $25^{\circ} \mathrm{C}$ vs sugar molal concentration. Sugar concentrations were determined gravimetrically. In addition, tables S3-6 show measured densities of sugar solutions at $21^{\circ} \mathrm{C}, 23^{\circ} \mathrm{C}$, and $25^{\circ} \mathrm{C}$.

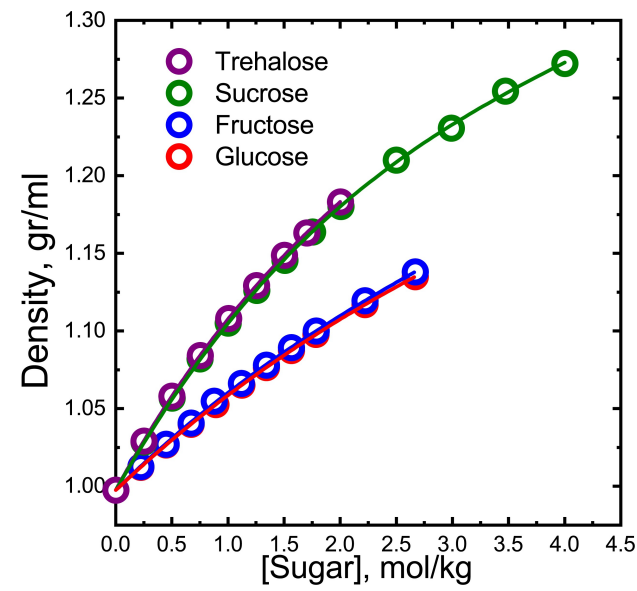

Figure S9. Densities of aqueous sugar solutions vs. sugar concentrations at $23^{\circ} \mathrm{C}$. The lines correspond to polynomial fits as follows:

$$
\begin{aligned}
& \rho_{\text {trehalose }}=0.99753+0.13306 \times m-0.025807 \times m^{2}+0.00283 \times m^{3} \\
& \rho_{\text {sucrose }}=0.99753+0.12667 \times m-0.02090 \times m^{2}+0.00161 \times m^{3} \\
& \rho_{\text {fructose }}=0.99753+0.0711 \times m-0.00926 \times m^{2}+0.00089 \times m^{3} \\
& \rho_{\text {glucose }}=0.99753+0.06827 \times m-0.00774 \times m^{2}+0.00055 \times m^{3}
\end{aligned}
$$

\begin{tabular}{|c|c|c|c|}
\hline \multirow[t]{2}{*}{$m / \mathrm{mol} \cdot \mathrm{kg}^{-1}$} & \multicolumn{3}{|l|}{$\rho / \mathrm{g} \cdot \mathrm{cm}^{-3}$} \\
\hline & $294.15 K$ & $296.15 K$ & $298.15 K$ \\
\hline 0 & 0.997982 & 0.997526 & 0.997027 \\
\hline 0.251 & 1.029862 & 1.029095 & 1.182238 \\
\hline 0.499 & 1.058665 & 1.058064 & 1.028371 \\
\hline 0.752 & 1.08498 & 1.084321 & 1.05743 \\
\hline 1.006 & 1.108791 & 1.108081 & 1.08363 \\
\hline 1.254 & 1.130062 & 1.129307 & 1.107342 \\
\hline 1.503 & 1.149636 & 1.148843 & 1.128526 \\
\hline 1.701 & 1.163987 & 1.163167 & 1.148023 \\
\hline 2.000 & 1.183975 & 1.183115 & 1.162324 \\
\hline
\end{tabular}

Table S3. Aqueous trehalose solution density. 
Table S4. Aqueous sucrose solution density.

\begin{tabular}{|c|c|c|c|}
\hline \multirow[t]{2}{*}{$m / \mathrm{mol} \cdot \mathrm{kg}^{-1}$} & \multicolumn{3}{|c|}{$\rho / \mathrm{g} \cdot \mathrm{cm}^{-3}$} \\
\hline & $294.15 K$ & $296.15 K$ & $298.15 K$ \\
\hline 0 & 0.997982 & 0.997526 & 0.997027 \\
\hline 0.250 & 1.029151 & 1.028625 & 1.028062 \\
\hline 0.502 & 1.057381 & 1.056793 & 1.056171 \\
\hline 0.751 & 1.082668 & 1.082026 & 1.081354 \\
\hline 1.000 & 1.105777 & 1.105088 & 1.104371 \\
\hline 1.255 & 1.27003 & 1.126273 & 1.125515 \\
\hline 1.506 & 1.146267 & 1.1455 & 1.144707 \\
\hline 1.752 & 1.64625 & 1.163829 & 1.163007 \\
\hline 2.005 & 1.80959 & 1.180146 & 1.179289 \\
\hline 2.499 & 1.210822 & 1.210098 & 1.209283 \\
\hline 2.989 & 1.231549 & 1.230711 & 1.229832 \\
\hline 3.472 & 1.255303 & 1.254484 & 1.253606 \\
\hline 4.000 & 1.273264 & 1.272288 & 1.271301 \\
\hline
\end{tabular}

Table S5. Aqueous fructose solution density.

\begin{tabular}{llll}
\hline $\mathrm{m} / \mathrm{mol} \cdot \mathrm{kg}^{-1}$ & $\rho / \mathrm{g} \cdot \mathrm{cm}^{-3}$ & & \\
\cline { 2 - 4 } & $294.15 \mathrm{~K}$ & $296.15 \mathrm{~K}$ & $298.15 \mathrm{~K}$ \\
\hline 0 & 0.997982 & 0.997526 & 0.997027 \\
0.224 & 1.013241 & 1.01273 & 1.01218 \\
0.447 & 1.027719 & 1.027162 & 1.02656 \\
0.670 & 1.041422 & 1.04082 & 1.040172 \\
0.877 & 1.055577 & 1.05493 & 1.054234 \\
1.118 & 1.067026 & 1.066341 & 1.065608 \\
1.341 & 1.078763 & 1.078046 & 1.077273 \\
1.564 & 1.090106 & 1.089354 & 1.088548 \\
1.784 & 1.100755 & 1.099975 & 1.099133 \\
2.220 & 1.120452 & 1.119614 & 1.118712 \\
2.667 & 1.139018 & 1.138129 & 1.13717 \\
\hline & & & \\
\hline
\end{tabular}


Table S6. Aqueous glucose solution density.

\begin{tabular}{llll}
\hline $\mathrm{m} / \mathrm{mol} \cdot \mathrm{kg}^{-1}$ & $\rho / \mathrm{g} \cdot \mathrm{cm}^{-3}$ & & \\
\cline { 2 - 4 } & $294.15 \mathrm{~K}$ & $296.15 \mathrm{~K}$ & $298.15 \mathrm{~K}$ \\
\hline 0 & 0.997982 & 0.997526 & 0.997027 \\
0.223 & 1.01287 & 1.012374 & 1.011839 \\
0.448 & 1.02718 & 1.026645 & 1.026075 \\
0.670 & 1.040554 & 1.039984 & 1.039381 \\
0.891 & 1.053245 & 1.052644 & 1.05201 \\
1.123 & 1.065804 & 1.065173 & 1.06451 \\
1.342 & 1.077153 & 1.076495 & 1.075806 \\
1.564 & 1.088125 & 1.087441 & 1.086728 \\
1.783 & 1.098531 & 1.097823 & 1.097088 \\
2.220 & 1.117755 & 1.117005 & 1.116231 \\
2.667 & 1.135773 & 1.134986 & 1.134176 \\
\hline & & & \\
\hline
\end{tabular}

\section{References}

(1) Lehmann, C. W.; Stowasser, F. The Crystal Structure of Anhydrous $\beta$-Caffeine as Determined from X-Ray Powder-Diffraction Data. Chem. - $A$ Eur. J. 2007, 13 (10), 2908-2911.

(2) Sutor, J. The Structures of the Pyrimidines and Purines. VII. The Crystal Structure of Caffeine. Acta Crystallogr. 1958, $11,453-458$.

(3) Sapir, L.; Harries, D. Origin of Enthalpic Depletion Forces. J. Phys. Chem. Lett. 2014, 5 (7), 1061-1065.

(4) Sapir, L.; Harries, D. Is the Depletion Force Entropic? Molecular Crowding beyond Steric Interactions. Curr. Opin. Colloid Interface Sci. 2015, 20 (1), 3-10.

(5) Lekkerkerker, H. N. W.; Tuinier, R. Colloids and the Depletion Interaction; Springer: Heidelberg, 2011; Vol. 833, pp 1-50.

(6) Asakura, S.; Oosawa, F. On Interaction between Two Bodies Immersed in a Solution of Macromolecules. J. Chem. Phys. 1954, 22 (7), 12551256.

(7) Asakura, S.; Oosawa, F. Interaction between Particles Suspended in Solutions of Macromolecules. J. Polym. Sci. 1958, 33 (126), 183-192.

(8) Eisenhaber, F.; Lijnzaad, P.; Argos, P.; Sander, C.; Scharf, M. The Double Cubic Lattice Method: Efficient Approaches to Numerical Integration of Surface Area and Volume and to Dot Surface Contouring of Molecular Assemblies. J. Comput. Chem. 1995, 16 (3), $273-284$.

(9) Moore, W. Physical Chemistry; Longman: London, 1972; pp 234-236.

(10) Harries, D.; Rösgen, J. A Practical Guide on How Osmolytes Modulate Macromolecular Properties. In Methods in Cell Biology; Correria, J. J., Detrich, H. W., Eds.; Elsevier: London, 2008; Vol. 84, pp 716-721.

(11) Kirkwood, B.; Buff, F. The Statistical Mechanical Theory of Solutions. I. J. Chem. Phys. 1951, 19, 774-777.

(12) Courtenay, E. S.; Capp, M. W.; Anderson, C. F.; Record, M. T. Vapor Pressure Osmometry Studies of Osmolyte-Protein Interactions: Implications for the Action of Osmoprotectants in Vivo and for the Interpretation of "osmotic Stress" Experiments in Vitro. Biochemistry 2000, 39 (15), 4455-4471.

(13) Parsegian, V. A. Protein-Water Interactions. Int. Rev. Cytol. 2002, 215, 1-31.

(14) Al-Maaieh, A.; Flanagan, D. R. Salt Effects on Caffeine Solubility, Distribution, and Self-Association. J. Pharm. Sci. 2002, 91 (4), 1000-1008.

(15) Cesàro, A.; Russo, E.; Tessarotto, D. Thermodynamics of Caffeine in Aqueous Denaturant Solutions. J. Solution Chem. 1980, 9 (3), $221-235$.

(16) Salvalaglio, M.; Perego, C.; Giberti, F.; Mazzotti, M.; Parrinello, M. Molecular-Dynamics Simulations of Urea Nucleation from Aqueous Solution. Proc. Natl. Acad. Sci. U. S. A. 2015, 112 (1), E6-E14. 